\title{
AHPMANAGER - A DECISION MAKING SUPPORT SYSTEM BASED ON THE AHP
}

\author{
Victoria Kusherbaeva ${ }^{1} *$, Yuriy Sushkov ${ }^{2}$, Gayk Tamazyan ${ }^{3}$ \\ Department of Mathematics \& Mechanics \\ Saint-Petersburg State University \\ St. Petersburg, Russia \\ Email $^{1}$ : kusher.v@gmail.com \\ E-mail'2:Yu.Sushkov@mail.ru \\ Email $^{3}$ : tamazyangayk@gmail.com
}

\begin{abstract}
A software package which implements the AHP is developed and presented. The program uses results obtained by the authors regarding scales and consistency measures. Three scales are suggested: the multiplicative Saaty scale, the additive Bruck scale and the logistic scale. Two alternative consistency measures based on cyclic triads are used along with the consistency ratio. The work of the program is demonstrated on the chess tournament problem.
\end{abstract}

Keywords: pairwise comparisons, scales, inconsistency, decision support, software

\section{Introduction}

Multiattribute problems containing qualitative criteria that cannot be exactly expressed in a numeric way are commonly encountered in different areas. To deal with such problems the analytic hierarchy process (AHP) which has been in the scope of the community for many years is often suggested.

The AHP is used in the great range of applications in different areas (Forman \& Gass, 2001). There are a number of programs that implement the AHP: Expert Choice (http://www.expertchoice.com), Decision Lens (http://www.decisionlens.com), Make It Rational (http://makeitrational.com), Select Pro (http://www.selectprosoftware), Criterion DecisionPlus (http://www.infoharvest.com). In this work we present another program that uses results obtained by the authors regarding scales and consistency measures. Moreover, special attention is given to the visual representation of the decision making process.

The structure of the article as follows. Section 2 reviews theoretical background of using alternative scales and consistency measures. In the third section the problem that is used for demonstrating features of the program is presented. Section 4 describes the process of solving a demo problem. Section 5 reviews additional features of the program. The final section summaries results.

\section{Alternative scales and consistency measures}

Before presenting the demo problem and features of the software package we briefly review alternative scales that are implemented in the program, theoretical facts about them, and two consistency measures. The detailed discussion on using alternative scales and consistency measures in the AHP can be found in (Kusherbaeva, Sushkov \& Tamazyan, 2011).

\footnotetext{
${ }^{*}$ Corresponding author
} 


\subsection{Alternative scales}

Following (Ji \& Jiang, 2002) scales are presented consisting of two parts: verbal and numerical ones. The verbal part contains qualitative gradations of relative importance and integer numbers $\lambda$ that present them (shown in Table 1). It is common for every scale under discussion.

Table 1. The verbal part of the scales.

\begin{tabular}{|c|c|c|c|c|c|c|}
\hline$\lambda$ & 0 & 2 & 4 & 6 & 8 & $1,3,5,7$ \\
\hline Semantics & equal & moderate & strong & very strong & extreme & $\begin{array}{c}\text { intermediate } \\
\text { values }\end{array}$ \\
\hline
\end{tabular}

A positive (negative) $\lambda$ assigned to the pair $\left(C_{i}, C_{j}\right)$ means that $C_{i}$ surpasses $C_{j}\left(C_{j}\right.$ surpasses $\left.C_{i}\right)$ with the grade that corresponds to semantics of $\lambda$.

Three scales are implemented in the program: Saaty scale, Bruck scale and the logistic scale. Following (Kusherbaeva \& Sushkov, 2010) we introduce the scales by defining mappings from the verbal part into the numeric one (shown in Table 2).

Table 2. The functions that define numerical parts of the scales.

\begin{tabular}{|l|c|l|}
\hline Scale name & Function that defines the numerical part & Scale parameters \\
\hline Saaty scale & $\phi_{S}(\lambda)=\left(x_{S}|\lambda|+1\right)^{\operatorname{sign} \lambda}$ & $x_{S}$ - scale parameter \\
\hline Bruck scale & $\varphi_{B}(\lambda)=c_{B}+\lambda x_{B}$ & $\begin{array}{l}c_{B} \text { - center parameter } \\
x_{B} \text { - scale parameter }\end{array}$ \\
\hline Logistic scale & $\varphi_{L}(\lambda)=2 /(1+\exp (-\mu \lambda))$ & $\mu$ - steepness parameter \\
\hline
\end{tabular}

Saaty scale is a generalized version of the fundamental one. Bruck proposed his scale in (Bruck \& Burkov, 1972). A logistic scale has been introduced in (Sushkov \& Kusherbaeva, 2010). Also we define classical parameters that correspond to the initial variants of the scales. Saaty scale with $x_{s}=1$ corresponds to the fundamental scale, for Bruck scale the classical case is $c_{B}=1, x_{B}=0.1$, for the logistic scale $\mu=1$.

\subsection{Consistency measures}

The consistency ratio cannot be used with Bruck and the logistic scales because they are not multiplicative. Following (Gass, 1998) two consistency measures based on cyclic triads are implemented in the program. The first consistency measure is a number of cyclic triads. The second one is a coefficient of consistency proposed in (Kendall \& Smith, 1940) that is called 'Kendall-Smith coefficient' in the program. Kendall-Smith coefficient can be used only if there are no equivalences among objects being compared.

\section{Chess tournament problem}

The second London Chess Classic tournament was held in December 2010. Eight chess Grandmasters participated in the tournament, results of their games are presented in Table 3 ( 3 scores for a victory, 1 score for a tie, 0 - for a loss). According to the sums of scores the winner is Magnus Carlsen, Luke McShane and Viswanathan Anand share the second place. 
Table 3. London Chess Classis 2010 standings.

\begin{tabular}{|l|l|c|c|c|c|c|c|c|c|c|}
\hline $\mathrm{Nr}$ & Name & 1 & 2 & 3 & 4 & 5 & 6 & 7 & 8 & $\Sigma$ \\
\hline 1 & Short, Nigel & & 0 & 1 & 0 & 0 & 1 & 0 & 0 & 2 \\
\hline 2 & McShane, Luke & 3 & & 1 & 1 & 1 & 1 & 3 & 1 & 11 \\
\hline 3 & Adams, Michael & 1 & 1 & & 1 & 1 & 3 & 0 & 1 & 8 \\
\hline 4 & Anand, Viswanathan & 3 & 1 & 1 & & 1 & 1 & 3 & 1 & 11 \\
\hline 5 & Nakamura, Hikaru & 3 & 1 & 1 & 1 & & 1 & 0 & 3 & 10 \\
\hline 6 & Howell, David & 1 & 1 & 0 & 1 & 1 & & 0 & 0 & 4 \\
\hline 7 & Carlsen, Magnus & 3 & 0 & 3 & 0 & 3 & 3 & & 1 & 13 \\
\hline 8 & Kramnik, Vladimir & 3 & 1 & 1 & 1 & 0 & 3 & 1 & & 10 \\
\hline
\end{tabular}

\section{Solving the demo problem}

\subsection{Building the hierarchy}

First of all the hierarchy that presents the problem must be specified in the program. The screenshot of the main window of the program with the hierarchy is shown in Figure 1.

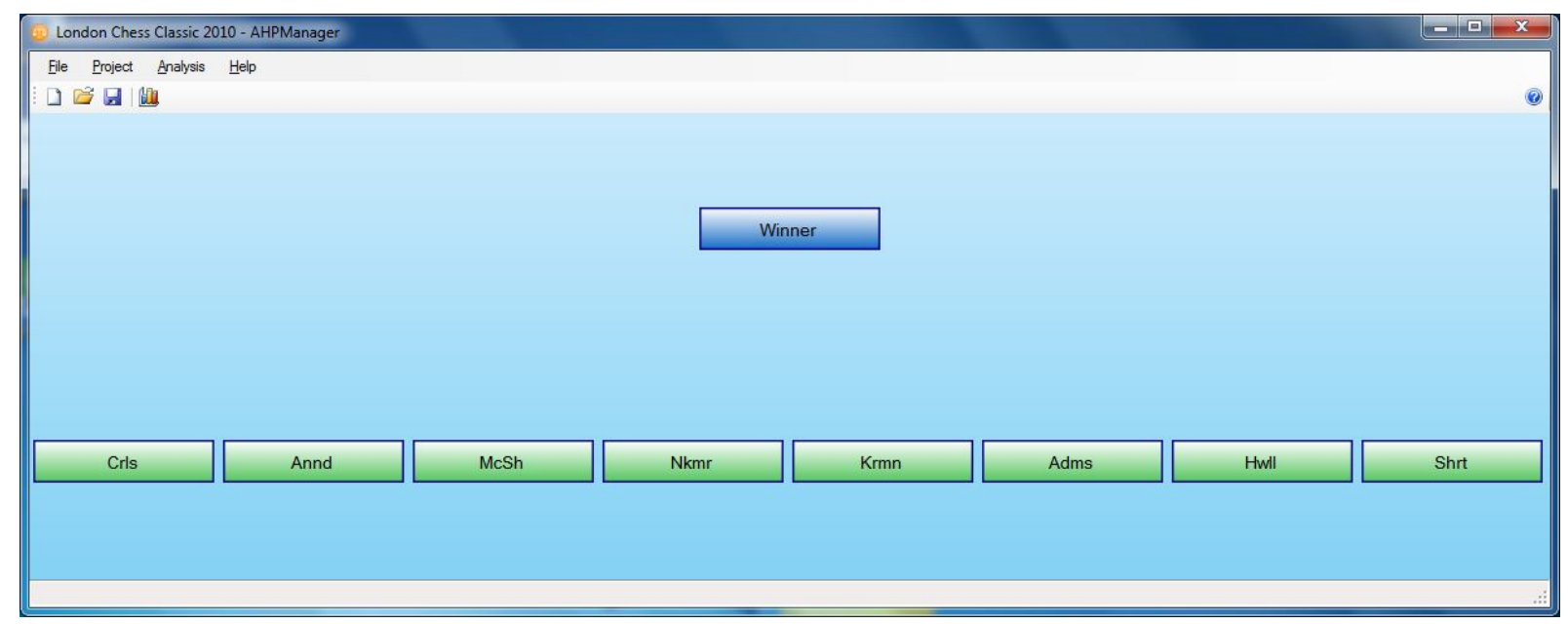

Figure 1. The main window of the program with the hierarchy of the chess tournament problem.

A user can modify the existing hierarchy: to add or remove elements and levels, or edit their properties (a title and a description). To do it one should right-click on the button that presents a hierarchy element and choose the corresponding item from the context menu (Figure 2). 

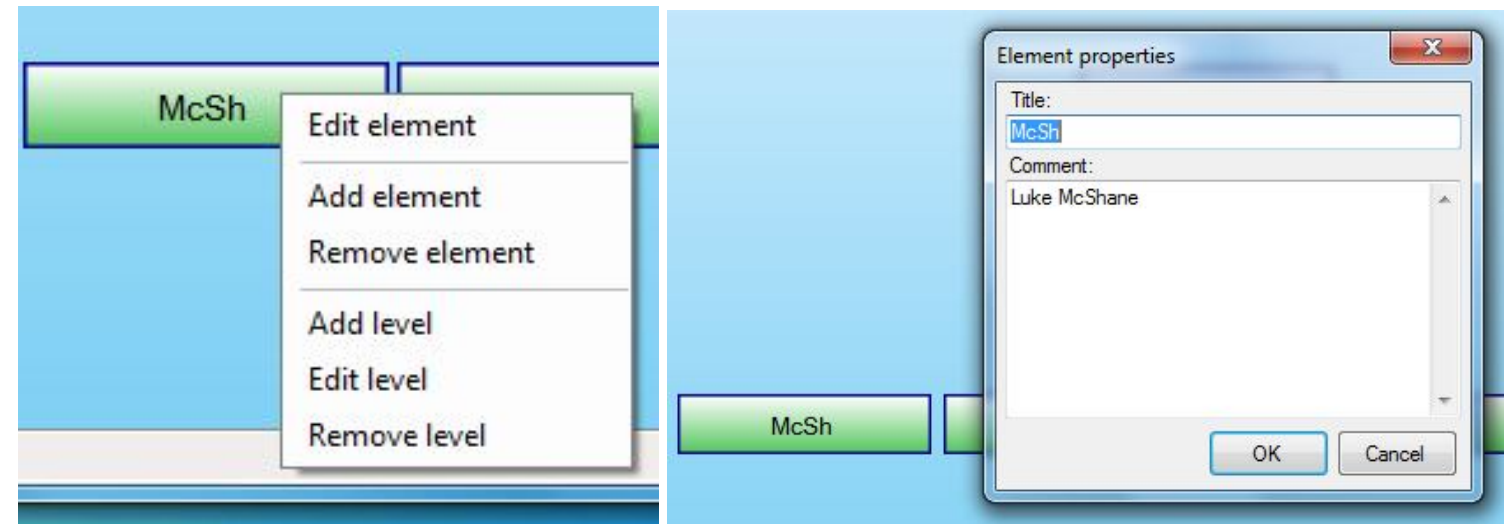

Figure 2. A context menu and a properties window of the hierarchy element.

\subsection{Comparing players}

To compare elements of the hierarchy according to the element on the higher level one should click the button that represents the element.
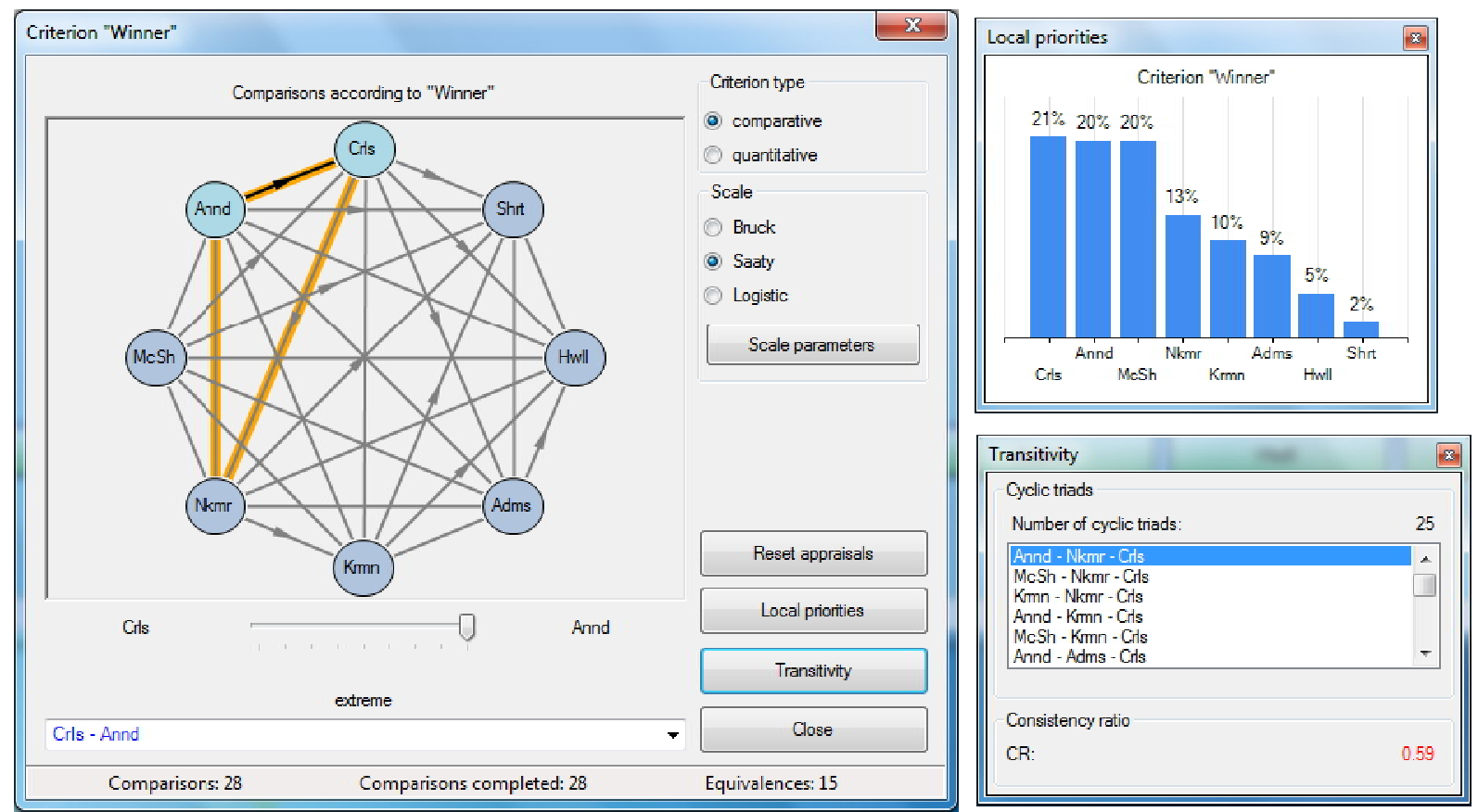

Figure 3. The pairwise comparisons window, the local priorities window, and the transitivity window.

In Figure 3 the comparisons window, the local priorities window and the transitivity window are shown. On the left side of the comparisons window a preference graph that represents pairwise comparisons made by a decision maker is located. Under it the slider to set results of pairwise comparisons and the falling list of pairs of elements are located. Also a user can choose a pair of elements by clicking the vertices of the preference graph.

There is also a possibility to set a scale type and scale parameters. Scale parameters window for Saaty scale is presented in Figure 4. 


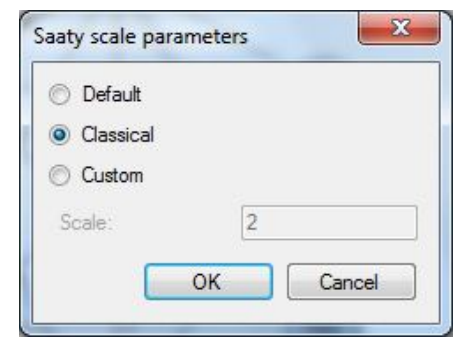

Figure 4. Scale parameters window for Saaty scale.

A scale and its parameters should be chosen depending on the problem. For example, following the properties of Saaty scale priorities of the best players get greater when $x_{s}$ increases. So, as it is shown in Figure 5, the first place should be shared between McShane and Anand, and Carlsen should be on the third place.

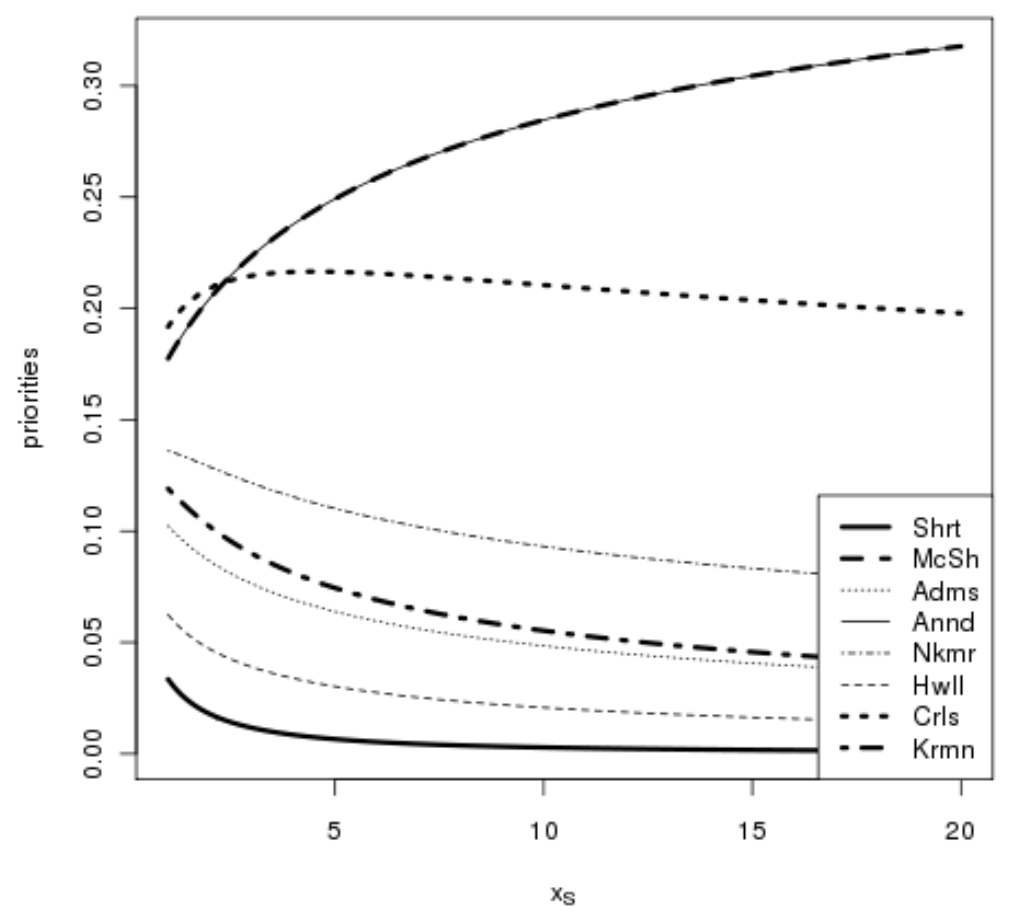

Figure 5. Priorities of the players gained using Saaty scale for different $x_{s}$ values.

The local priorities window shows the priorities of the elements being compared depending on the appraisals that have been set by a decision maker. The diagram and the priority values change depending on appraisals, scale type or scale parameters. Thus, a decision maker can conveniently determine a scale and its parameters for a particular problem.

Two measures of consistency are shown in the transitivity window: number of cyclic triads and consistency ratio (Saaty, 1980). Consistency ratio appears only if Saaty scale with the classical parameter is used. The third measure - Kendall-Smith coefficient - appears only if there are no missing appraisals of pairwise comparisons and no equivalences among them. There have been ties among the games so the Kendall-Smith coefficient is missing in Figure 3. 
Also a user can highlight a cyclic triad by choosing it from the falling list of alternatives. The example is shown in Figure 3: the cyclic triad 'Anand - Nakamura - Carlsen' is selected.

\section{Additional features}

Due to limited size of the paper we do not describe in detail other features of the program and only list them:

- support of quantitative criteria,

- display statistics of the hierarchy,

- saving and loading projects to the XML-based format,

- presenting results of solving the problem as a report,

- exporting the report to PDF and Excel formats.

\section{Conclusion}

In this paper we have described a decision making support system based on the AHP. The presented program implements theoretical results obtained by the authors (Kusherbaeva, Sushkov \& Tamazyan, 2011). It has a user-friendly interface and provides visualization of the decision-making process and possibility to choose a scale and its parameters for every element of the hierarchy. Also a decision maker can highlight cyclic triads and view intermediate results (local priorities).

\section{REFERENCES}

Bruck, B.N., \& Burkov V.N. (1972). Methods of expert estimation in objects ranging problems (in Russia). Proceedings of the Academy of Sciences of the USSR. Engineering Cybernetics, 3, 29-39.

Forman, E.H., \& Gass, S.I. (2001). The Analytic Hierarchy Process: An Exposition. Operation Research, 49, 469-486.

Gass, S.I. (1998). Tournaments, transitivity and pairwise comparison matrices. Journal of the Operational Research Society, 49, 616-624.

Ji, P., \& Jiang, R. (2002). Scale transitivity in the AHP. Journal of the Operational Research Society, 54, 896-905.

Kendall, M., \& Smith, B. (1940). On the method of pair comparisons. Biometrika, 31, 324-345.

Kusherbaeva, V.T., \& Sushkov, Yu.A. (2010). Scales and their properties in the AHP (in Russian). Journal of the KBSC of the Academy of Sciences of Russia, 37.

Kusherbaeva, V., Sushkov, Yu., \& Tamazyan, G. (2011). Comparative study of scales and consistency measures in the AHP. Proceedings of the 11th ISAHP; Italy, Sorrento, June 15-18, 2011.

Saaty, T.L. (1980). The analytic hierarchy process. McGraw-Hill. 